\title{
Passive control of jet flows using lobed nozzle geometries
}

\author{
Ilinca Nastase a and Amina Meslem \\ LEPTAB, University of La Rochelle, Avenue Michel Crépeau 17042 La Rochelle Cedex 01, France
}

Received 1st September 2006, accepted 23 January 2007

\begin{abstract}
Passive control of the entrainment phenomenon in the turbulent jets is a mixing enhancement economical method of wide interest in the industrial field. A lobed nozzle without lobes inclinations allows improving mixing in the generated flow compared to a reference circular jet. A second lobed nozzle, having the same exit plane geometry but with inclined lobes intensifies, in a considerable manner, the entrainment which reaches up to four times the one in the reference circular jet. The lobed jets vortical dynamics analysis shows that the azimuthal structures are not annular like in the case of a circular jet, but discontinuous, due to the shear of the transverse flow induced by the curvature variation of the exit plane. The streamwise structures development at the discontinuities locations is probably explaining the entrainment benefit observed in the lobed jet without inclination angles. The lobed jet issued from the second nozzle presents like the first lobed jet, discontinuous azimuthal structures, but its remarkable induction benefit is not merely owed to the previous phenomenon. The intensification and organisation of the streamwise vorticity field into large scale structures, resulting in a consequent mixing enhancement, are connected to the increased shear produced by the lobes inclinations.
\end{abstract}

Key words: Passive control / lobed nozzle / turbulent jet / vortex / mixing

Résumé - Contrôle passif des jets par des buses de soufflage à géométries lobées. Le contrôle passif de l'auto-induction des jets turbulents est un moyen de mélange peu coûteux qui intéresse un large domaine industriel. Une buse lobée droite, permet d'améliorer le mélange du jet qui en est issu, par rapport à un jet circulaire de référence. Une seconde buse lobée de même plan de soufflage et à lobes inclinés, intensifie de façon considérable l'auto-induction qui atteint jusqu'à quatre fois celle du jet circulaire de référence. L'analyse de la dynamique tourbillonnaire, montre que les structures azimutales des jets lobés ne sont pas annulaires comme dans un jet circulaire, mais discontinues en raison du cisaillement de l'écoulement transversal généré par la variation du rayon de courbure du plan de soufflage. L'épanouissement des structures longitudinales aux points de discontinuités explique probablement le gain d'induction observé dans le jet lobé droit. Le jet lobé issu de la buse à frontière inclinée, présente comme le précédent des structures azimutales discontinues mais le gain d'induction important qui le caractérise n'est pas dû uniquement à ce phénomène. L'intensification et l'organisation du champ de vorticité longitudinale en structures de grande échelle, conduisant à une intensification conséquente du mélange, sont liées à l'accroissement du cisaillement provoqué par les inclinaisons.

Mots clés : Contrôle passif / buse lobée / jet turbulent / vortex/ mélange

\section{Introduction}

Active and passive control of jet flows has been widely used to improve combustion efficiency and to reduce pollutants in mixing chambers or to attenuate ejectors infrared and noise radiation in aircraft. Active means use controlled acoustical excitation which favors under certain conditions the appearance of large scale structures

${ }^{a}$ Corresponding author: inastase@univ-lr.fr with high energy levels that are responsible for mixing [1-3]. Less expensive and easier to apply, passive methods [4-7] consist of using three dimensional nozzles with geometries that became progressively complex in the past decades $[8,9]$ in order to enhance the self-induction of asymmetric coherent structures and it has been suggested in recent work of $\mathrm{Hu}$ et al. $[10,11]$, that the lobed nozzle is an extraordinary mixing device.

Even though the authors are thereby generally qualifying the lobed nozzles, only one geometry was tested. 

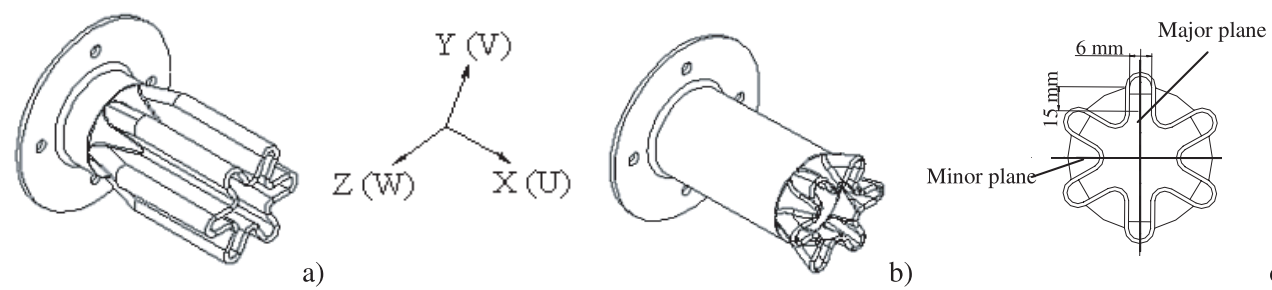

b)

c)

Fig. 1. a) Lobed nozzle 1, b) lobed nozzle 2, c) view of the lobed geometry in the $(Y Z)$ exit plane.

The diffuser nozzle is lobed shaped with 6 lobes having respectively the angle between the $X$ axis and the tangent at the lobe peaks $14^{\circ}$, and the angle between the $X$ axis and the tangent at the troughs $22^{\circ}$. The exploration of the first three equivalent diameters of their lobed jet was based on the analysis of the mean and instantaneous vorticity fields and of the mean turbulent kinetic energy fields. This analysis revealed the existence of a pair of large scale streamwise vortices at every lobe peak which dominate the spreading and mixing enhancement phenomena. These observations are promising for the mixing performance which might be offered by the lobed jet in its near field. However, a quantification of the entrainment flow rate is missing and therefore, a comparison with a jet having an axisymmetric geometry or other asymmetric geometry is not achievable. Moreover, through the results presented by the authors, it is not possible to discern the part played by the lobe deflection angles from the one played by the lobed geometry of the exit plane.

The present study could be placed in the continuity of the previous works. Its main objective is to quantify and to compare the mixing performance of two lobed jets with the one of a reference axisymmetric jet having the same exit diameter and initial volume rate. The first lobed jet is issued from a lobed nozzle without lobe inclination angles. The second studied nozzle has the same geometry as the one proposed by $\mathrm{Hu}$ et al. [10]. Through this study, we aimed at assessing the role played by the lobe inclination angles in the spreading and mixing enhancement phenomena. This way, a vortical dynamics analysis of the explored lobed jets compared to the reference circular jet attempts in highlighting the lobed jet mixing enhancement governing phenomena.

\section{Experimental set-up and exit conditions}

Turbulent isothermal air jets issued from a circular reference nozzle having the diameter $D=40 \mathrm{~mm}$ and from two lobed nozzles, respectively called lobed nozzle 1 (Fig. 1a) and lobed nozzle 2 (Fig. 1b), having the same equivalent diameter $D_{\mathrm{e}}=40 \mathrm{~mm}$ and the same exit plane geometry (Fig. 1c), were investigated within an axial distance of $X=5 D_{\mathrm{e}}$. The width of each lobe is $6 \mathrm{~mm}$ and its height is $15 \mathrm{~mm}$. The lobed nozzle 2 has exactly the same geometry as the one studied by $\mathrm{Hu}$ et al. [10]. This nozzle is built-up from a circular section tube of $40 \mathrm{~mm}$ diameter and $76.5 \mathrm{~mm}$ length which is connected to a shorter lobed geometry. The inner penetration angle of the lobe troughs between the $X$ axis and the tangent at the troughs is $\alpha_{\text {in }}=22^{\circ}$, and the outer penetration angle of the lobe peaks between the $X$ axis and the tangent at the lobe peaks is $\alpha_{\text {out }}=14^{\circ}$. Our wish being to impose $\alpha_{\text {in }}=0$ and $\alpha_{\text {out }}=0$ for the lobed nozzle 1, the exit plane geometry is conserved on a length of $56.3 \mathrm{~mm}$. The resulting lobed tube is connected through a lobed divergent to a circular tube of $20.6 \mathrm{~mm}$ length.

The air jet experimental facility consists of a centrifuge fan connected to a metallic pipe having $40 \mathrm{~mm}$ in diameter and $1 \mathrm{~m}$ in length. A honeycomb was placed upstream the nozzle at the end of the pipe, in order to reduce the turbulence level at the nozzle exit.

In a first time, the mean dynamic analysis of the three flows and the quantification of their entrained volume flow rates were based on two-dimensional Laser Doppler Anemometry (LDA) mean velocity fields measurements. The LDA system has two solids lasers: one $\mathrm{Nd}$ : Yag of $25 \mathrm{~mW}$, providing a monochromatic green beam $(514.5 \mathrm{~nm})$ and one Sapphire of $22 \mathrm{~mW}$ providing monochromatic blue beam (488 nm). The sampling time during the measurements was in the range of 60 to $100 \mathrm{~s}$ and the mean data rate from 0.5 to $4.8 \mathrm{kHz}$ depending on the flow velocity at the measurement point. The measuring volume at the lasers beam intersection is $0.04 \times 0.045 \times 0.378 \mathrm{~mm}$ large. The probe was mounted on a three-dimensional traverse system having the movement resolution and reproducibility of $6.25 \mu \mathrm{m}$. The distance between two measurement points was $2 \mathrm{~mm}$.

On the other hand, Particle Image Velocimetry (PIV) was employed to acquire instantaneous spatial distribution of the in-plane velocity. The PIV Dantec system was composed of a high resolution Kodak Megaplus ES1 CCD camera of $10^{6}$ pixels and a NewWave Pegasus of $150 \mathrm{~mJ}$ laser. The acquisition frequency of the PIV system was on the order of $5 \mathrm{~Hz}$. It should be noted that this frequency is much too reduced compared to the vortical structures shedding frequencies. In order to overcome this lack of information, the experimental campaign was completed with high speed visualizations using a CCD Nanosense MKII camera and a 4 W Nanopower laser providing a $795 \mathrm{~nm}$ laser sheet. The acquisition frequency of the visualization system could attain $5 \mathrm{kHz}$ for a window of $512 \times 512$ pixels. The experimental campaign was also completed with the recordings of the streamwise velocity signal $u(t)$ in the jet flows shear layers with a 55R03 Dantec fiber film running in the constant temperature mode.

Table 1 provides the exit conditions for the three nozzles. The initial volumetric flow rate $Q_{0}$ based on 
Table 1. Exit conditions of the jets.

\begin{tabular}{cccccccccc}
\hline $\begin{array}{c}\text { Nozzle } \\
\text { geometry }\end{array}$ & $Q_{0}\left(\mathrm{~m}^{3} \cdot \mathrm{s}^{-1}\right)$ & $U_{0}\left(\mathrm{~m}_{\mathrm{s}}{ }^{-1}\right)$ & $U_{\mathrm{OC}}\left(\mathrm{m} . \mathrm{s}^{-1}\right)$ & $R e_{0}$ & $R e_{0 \mathrm{C}}$ & $\theta_{0}(\mathrm{~mm})$ & \multicolumn{2}{c}{$f_{n}(\mathrm{~Hz})$} \\
\hline Circular & $4.82 \times 10^{-3}$ & 3.84 & 4.88 & 9785 & 12400 & 2.04 & & - \\
6-lobe 1 & $4.75 \times 10^{-3}$ & 3.78 & 5.82 & 9600 & 14800 & 1.43 & 1.38 & 168 & 224 \\
6-lobe 2 & $4.70 \times 10^{-3}$ & 3.74 & 5.89 & 9520 & 15000 & 3.95 & 2.18 & 343 & 470 \\
\hline
\end{tabular}

the mean velocity field at the nozzle exit was $4.82 \times$ $10^{-3} \mathrm{~m}^{3} \cdot \mathrm{s}^{-1}$ for the circular jet and respectively $4.75 \times$ $10^{-3} \mathrm{~m}^{3} \cdot \mathrm{s}^{-1}$ for the lobed jet 1 and $4.70 \times 10^{-3} \mathrm{~m}^{3} \cdot \mathrm{s}^{-1}$ for the lobed jet 2 . This could be traduced by a relative small deviation of the order of $2.5 \%$. As it can be observed in Table 1, the two lobed jets are accelerated at their exit plane showing a core velocity $U_{0 \mathrm{C}}=5.82 \mathrm{~m}^{3} \cdot \mathrm{s}^{-1}$ for the lobed jet 1 and $U_{0 \mathrm{C}}=5.89 \mathrm{~m}^{3} \cdot \mathrm{s}^{-1}$ for the lobed jet 2 . These velocities are to be compared to the circular jet centerline exit velocity $U_{0 \mathrm{C}}=4.88 \mathrm{~m}^{3} \cdot \mathrm{s}^{-1}$. The difference between the mean exit velocity $U_{0}$ and the centerline exit velocity $U_{0 \mathrm{C}}$, brings us to define two initial Reynolds numbers for each nozzle. The first one, noted as $R e_{0}$, is based on the mean exit velocity and it is almost equal for all three jets. The second one, noted $R e_{0 \mathrm{C}}$, is based on the centerline exit velocity and exhibits different values for each jet.

\section{Results and discussion}

An analysis of the spreading and spatial deformation of the lobed jets in comparison to the reference circular jet is first proposed in Figure 2 where we superposed their streamwise velocity contour $U / U_{0}=0.1$. It could be observed that the extremely intense lobe signature at $X=0.25 D_{\mathrm{e}}$ (Fig. 2a) tends to disappear at $X=3 D_{\mathrm{e}}$ (Fig. 2d), distance from which one could easily compare the spreading of the three jets. If it is obvious that the dynamical expansion of the lobed jet 2 is greater than the one of the circular jet, conversely, the lobed jet 1 presents a slightly larger, even comparable, expansion to the one of the circular jet.

The benefit in terms of mixing enhancement for the lobed jet 2 compared to the lobed jet 1 is confirmed in Figure 3a showing the streamwise evolutions of the normalized entrained flow rates of the lobed jets. In the initial region, the entrainment in the lobed jet 2 reaches up to four times the one in the reference circular jet and at $X=5 D_{\mathrm{e}}$ it is 2.5 times greater. As for the lobed jet 1 , even if its self-induction is superior to the one of the reference circular jet, the gain in entrainment is much reduced attaining a maximum of $70 \%$ at $X=1 D_{\mathrm{e}}$.

These results permit to quantify for the lobed jet, the importance of the role played in the mixing enhancement by the lobe inclination angles comparatively to the lobed geometry of the exit plane. The streamwise velocity decays presented in Figure 3b are in agreement with the previous results and allow the estimation of each jet potential core which is respectively $4 D_{\mathrm{e}}$ for the circular jet, $3 D_{\mathrm{e}}$ for the lobed jet 1 and $2 D_{\mathrm{e}}$ for the lobed jet 2 .
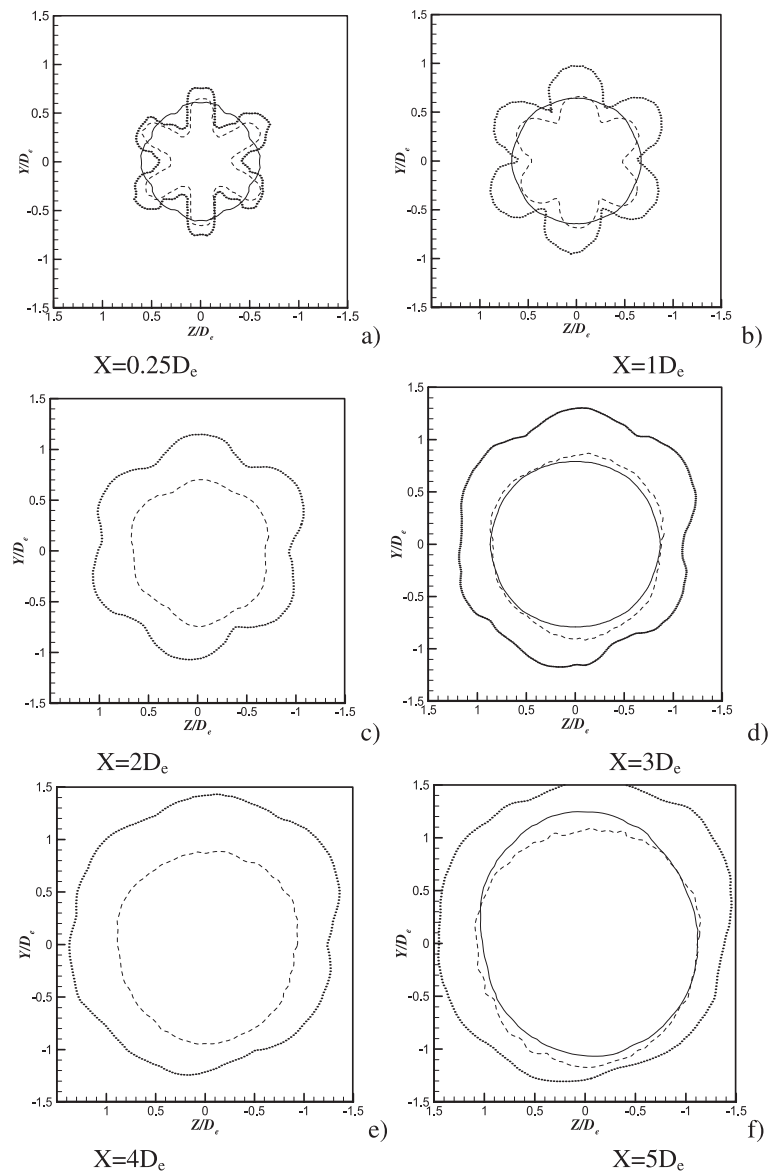

Fig. 2. Normalized streamwise velocity contour $U / U_{0}=0.1$ in the transverse $(Y Z)$ planes (solid - circular jet, dashed lobed jet 1 , dotted - lobed jet 2$)$.

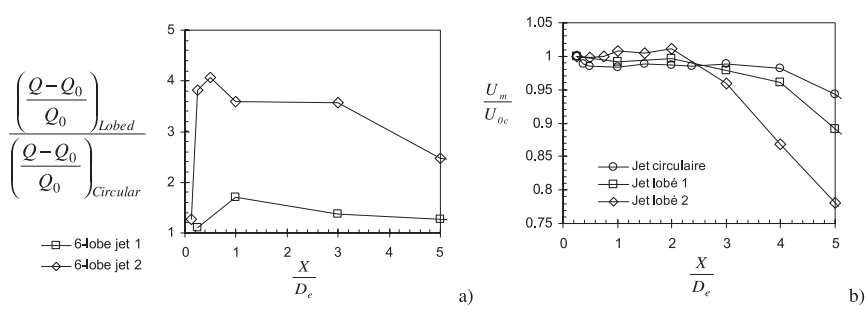

Fig. 3. a) Streamwise evolution of the normalized flow rate, b) streamwise evolution of the normalized axial velocity.

The momentum thickness distribution in the transverse planes $X=0.25 D_{\mathrm{e}}$ and $X=5 D_{\mathrm{e}}$ (Fig. 4 ) should bring along an indication on the lobed jets flow spreading and radial momentum transfer capability. For the circular jet (Figs. 4, 1a, 2a) the momentum thickness distribution is almost axisymmetric, showing a nearly equal 


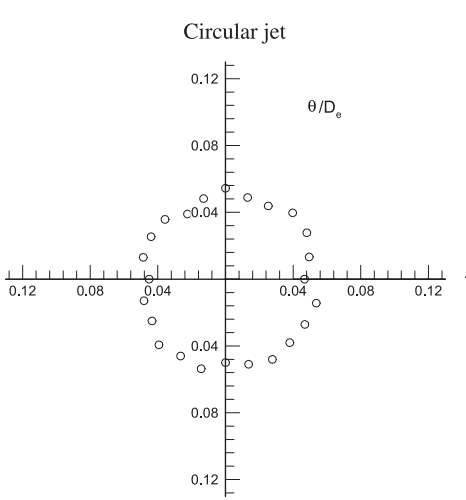

1a)

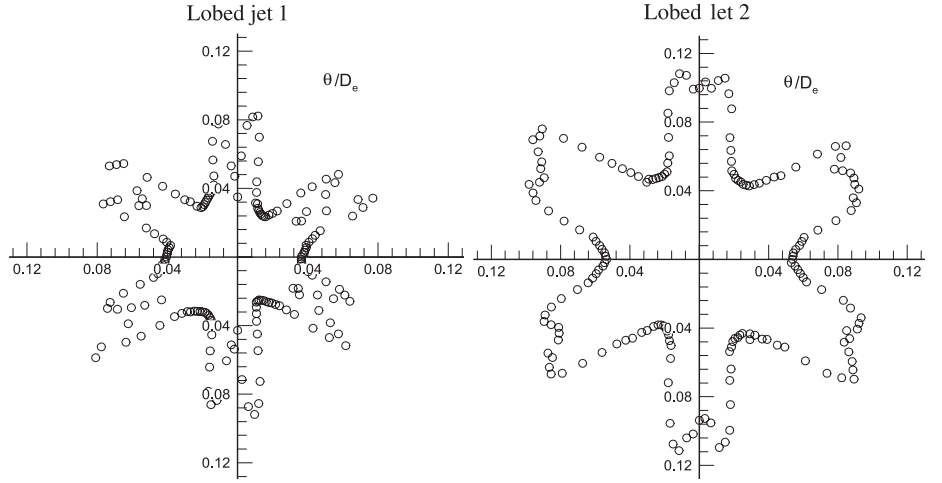

1b) 1c)

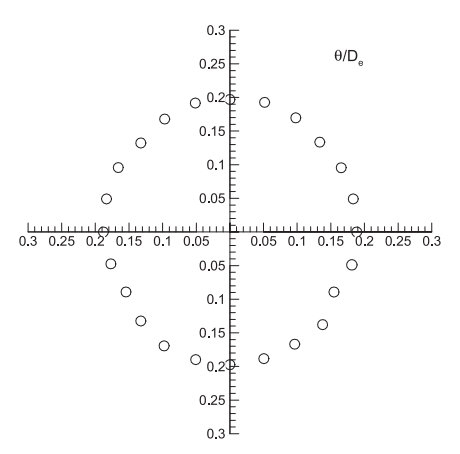

2a)

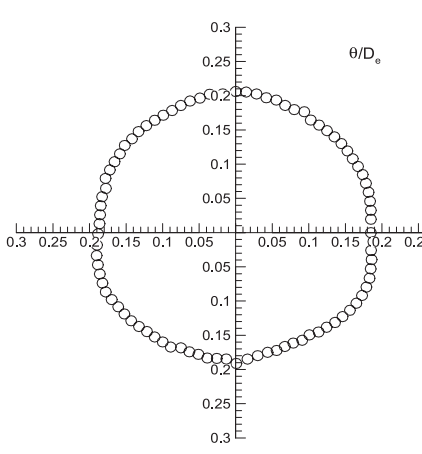

2b)

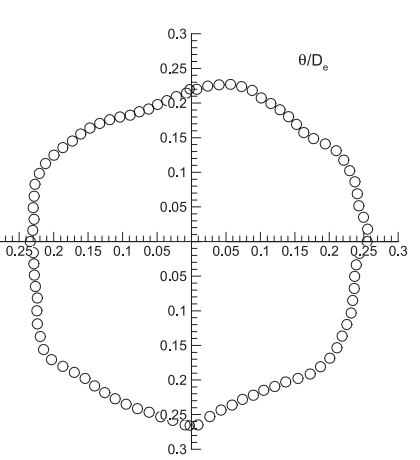

2c)

Fig. 4. Peripheral momentum thickness distribution in the transverse $(Y Z)$ planes: $(1) X=0.25 D_{\mathrm{e}},(2) X=5 D_{\mathrm{e}}$.

momentum transfer potential in all directions. It follows an entrainment velocity $U_{r}$ nearly constant (Fig. 5a). This result is totally predictable due to the nozzle circular geometry.

For the lobed jets (Figs. 4, 1b, 1c, 2b, 2c) the momentum thickness varies with the radial direction at the exit plane (Figs. 4, 1b, 1c) and its maximal values are not located in the major planes. These maxima are situated at the locations where it takes place a change in the sign of the radial velocity $U_{r}$ of the transverse flow (Figs. 5b and c). The latter is positive at the lobe peaks and negative at the lobe troughs showing an expansion of the flow in the lobes regions and an entrainment in the troughs regions. The value of $\theta_{\max } / D_{\mathrm{e}}$ was found to be 0.09 for the lobed jet 1 and 0.12 for the lobed jet 2 comparatively to 0.06 for the circular jet which is translated by a ratio of respectively 1.5 and 2 . As for the mean values, $\theta_{\text {mean }} / D_{\mathrm{e}}$ was found 0.06 for the lobed jet 1 and 0.08 for the lobed jet 2 to compare with 0.05 for the circular jet.

On the radial momentum thickness distribution in the farther field at $X=5 D_{\mathrm{e}}$, if for the lobed jet 1 one could note a quasi axisymmetric distribution (Figs. 4, 2b), this is not the case for the lobed jet 2 which still displays a slight asymmetry (Figs. 4, 2c). At this distance, the mean value $\theta_{\text {mean }} / D_{\mathrm{e}}$ is 0.19 for the circular jet, 0.20 for the lobed jet 1 and 0.26 for the lobed jet 2 . Thus, the superior entrainment capability previously observed for the lobed jet 2 , is in agreement with its larger momentum transfer capability.
After this analysis of the mean flows and their mixing capabilities, we attempt to elucidate hereafter, the lobed jet mixing enhancement governing mechanisms based on the instantaneous streamwise and transverse velocity fields as well as on the streamwise velocity spectra acquired in the shear layers. It appears (Figs. 6a and Figs. 7, 1a) that the azimuthal rings in the circular jet are not shed at a preferred frequency like it might be the case of a forced jet [12], or of a circular jet at lower Reynolds number, as we could note for $R e_{0} \approx 2500$ (Figs. 7, 2a, Tab. 2).

Oppositely, for the lobed jets, clearly detached preferred frequencies are visible on their spectra (Figs. 7, 1b and 1c) being two times higher for the lobed jet 2 (Tab. 1). Moreover, these frequencies are different if they are measured at the lobe troughs (D point) or at the lobe peaks (E point) with a ratio (in the previous order) of respectively 1.33 for the lobed jet 1 and 1.37 for the lobed jet 2 . At a lower Reynolds number $\left(R e_{0} \approx 2500\right)$ we could note that the previous phenomenon is conserved and the frequencies ratios are close to the ones above (Tab. 2). This result highlights a discontinuity of the azimuthal structures in the lobed jet which has been confirmed by the high speed visualisations. The azimuthal structures shedding at the lobe troughs are visible on the PIV images of the minor planes (Fig. 6b). The PIV images of the major planes being unfortunately incomplete (Fig. 6c), we present in Figure 6d, high speed visualisation images of the same planes. Close to the jet exit, the interior darker 

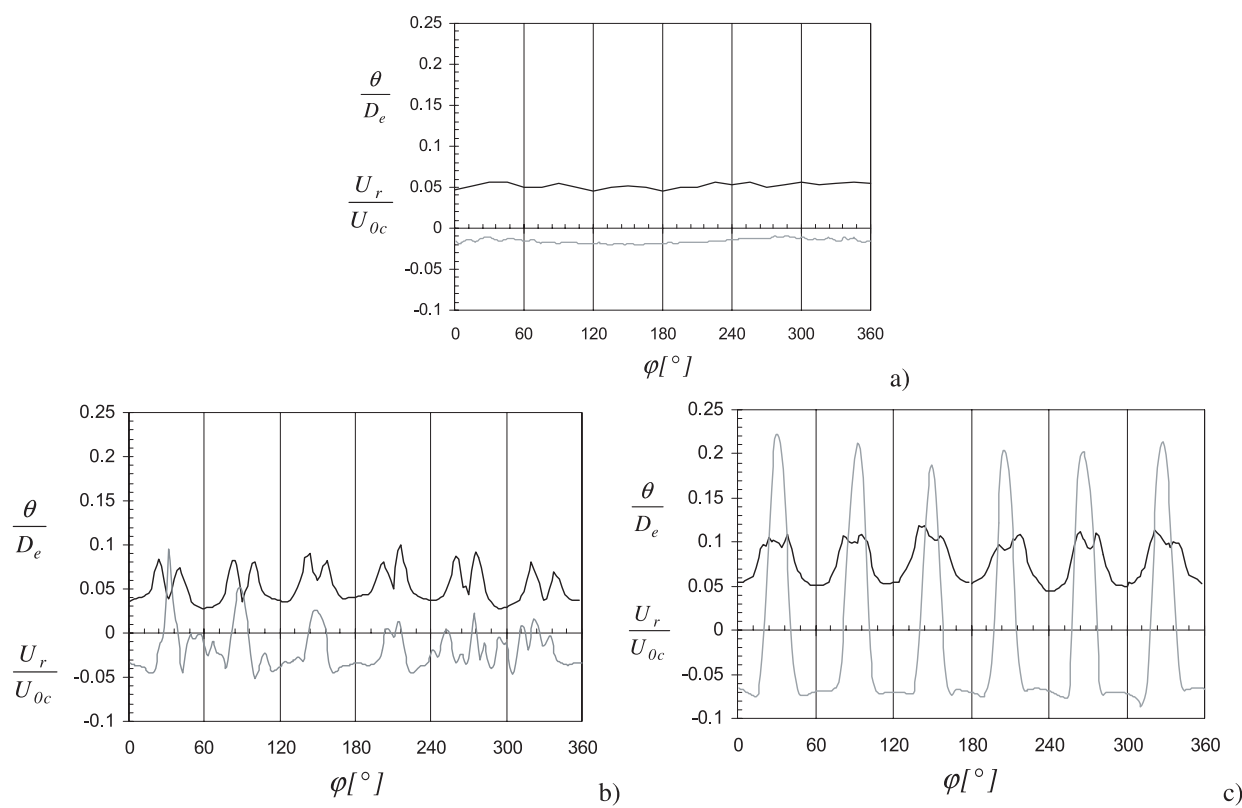

Fig. 5. Mean radial velocity at $r=0.60 D_{\mathrm{e}}$ and momentum thickness distribution in the transverse $(Y Z)$ plane at $X=0.25 D_{\mathrm{e}}$ : a) circular jet, b) lobed jet 1 , c) lobed jet 2 .

regions that could be observed are corresponding to the azimuthal structures detaching at the lobe sides and are perfectly visible on the images in Figure 6e. Their shedding frequencies are detected at the E point location on the streamwise velocity spectra presented in Figures $7,1 \mathrm{~b}$ and $7,1 \mathrm{c}$. The discontinuity of the azimuthal structures in the lobed jet is probably explained by the shear of its transverse flow (Figs. 5b and 5c), generated by the curvature radius variation on the lobed nozzle exit plane.

Being in the possession of valid PIV fields in the streamwise minor planes we wanted to compare the previous visual detection of the azimuthal structures, to a so called objective definition suggested by Jeong \& Hussain [13]. This method takes into account the positions of local pressure minima in the plane, excepted the ones due to the unsteady irrotational straining and to the viscous effects. Hence the presence of a local pressure minimum in the plane corresponds to a negative second eigenvalue $\lambda_{2}$ of the tensor $S^{2}+\Omega^{2}, S$ and $\Omega$ being respectively the symmetric and the antisymmetric parts of the velocity gradient tensor.

In our case, for PIV-2D measurements, only two components of three are acquired in the plane. This way, in the streamwise plane $(X Z)$ the $\partial v / \partial Y$ term is deduced from the continuity equation for an incompressible fluid. As a matter of fact, a symmetry of the flow relatively to the chosen median plane is admitted which enables us to consider as negligible the terms $\partial u / \partial Y$ and $\partial w / \partial Y$. Moreover, if we assume that the vertical component $v$ of the velocity is null in the considered plane, $\partial v / \partial X$ and $\partial v / \partial Z$ will also be null. However it should be noted that the previous assumptions are not acceptable for another streamwise plane parallel to the median plane.

This definition was applied to the three instantaneous fields presented in Figures $6 \mathrm{a}$ and $6 \mathrm{~b}$, of the three studied flows, and the results are presented in Figures 8, 1a, 1b and 1c. We show in Figures 8, 2a, 2b and $2 \mathrm{c}$, the corresponding normalized vorticity fields $\omega_{y}=$ $\frac{D_{\mathrm{e}}}{D_{\mathrm{cc}}}\left(\frac{\partial u}{\partial Z}-\frac{\partial w}{\partial X}\right)$. It is interesting to note that the $\lambda_{2}<0$ condition is more powerful than the vorticity fields to locate the vortical structures despite all the previously made assumptions. The corresponding $\omega_{Y}$ maximal magnitudes levels are respectively 1.8 times for the lobed jet 1 and 2.4 times for the lobed jet 2 greater than for the circular jet.

As well in the circular jet as in the lobed jet, the azimuthal vortices appear due to the shear between the jet flow and its ambience. Indeed in the $(X Z)$ plane of each jet a very good correlation between the instantaneous fields of the in-plane shear $\sigma_{X Z}=\frac{D_{\mathrm{e}}}{D_{0 c}}\left(\frac{\partial w}{\partial Z}-\frac{\partial u}{\partial Z}\right)$ and of the azimuthal vorticity $\omega_{Y}=\frac{D_{\mathrm{e}}}{D_{0 \mathrm{c}}}\left(\frac{\partial u}{\partial Z}-\frac{\partial w}{\partial X}\right)$ is obtained (Figs. 8, 3a-c).

In the circular jet, these vortices have an annular shape and their shedding is generating azimuthal instabilities at the origin of streamwise structures whose number is depending on the initial Reynolds number [14]. These streamwise structures which born on the braid (Figs. 9, 1a) between two azimuthal vortex rings which attenuate them as they are passing by, play a major role as suggested by Liepmann \& Gharib [14] in the selfinduction phenomenon of the circular jet. This type of vortices is also appearing in the lobed jets (Figs. 9, 1b and 1c) but they are much more developed at the same axial distance in the lobed jet 2. For this jet (Figs. 9, 1c) it is interesting to note the coexistence of streamwise and azimuthal structures owing to the previously noted azimuthal vortices discontinuities. This is not visible for the lobed jet 1 because the streamwise structures are not sufficiently developed at this distance. 


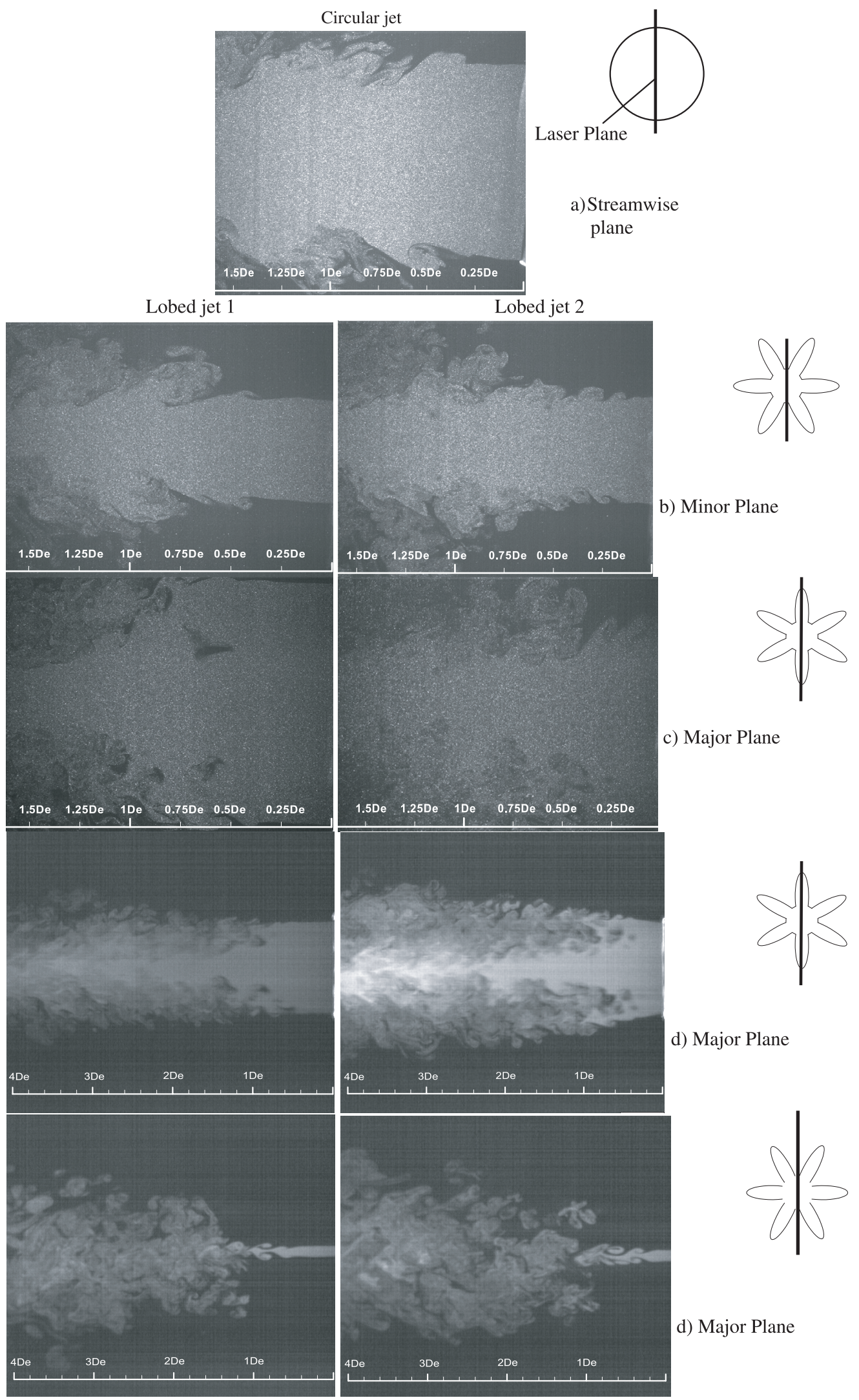

Fig. 6. a-c) PIV images, d) high speed visualization images. 

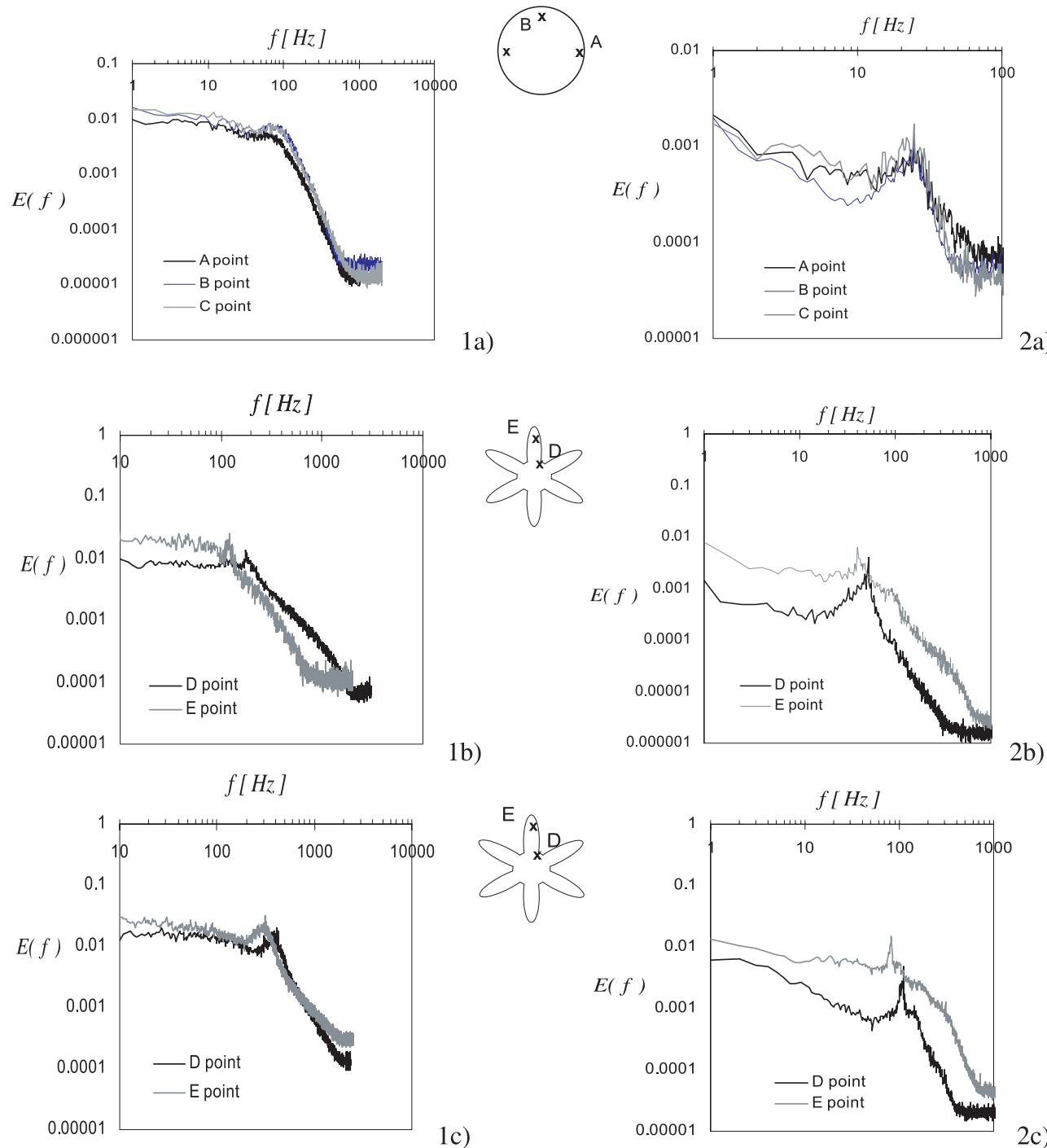

b)

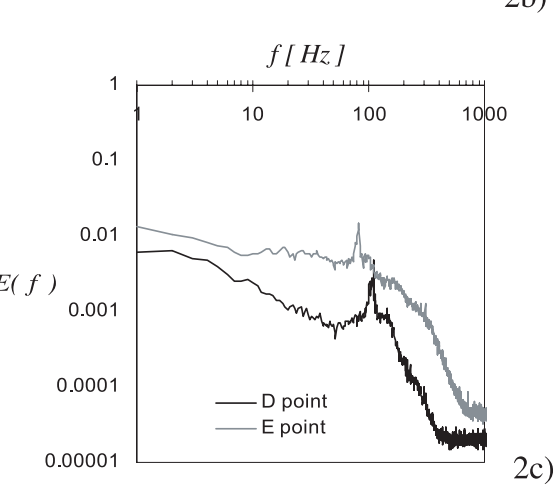

Fig. 7. Streamwise velocity spectra in the shear layer at $X=0.25 D_{\mathrm{e}}$ : (1) for $R e_{0} \sim 9600$, (2) for $R e_{0} \sim 2500$; a) circular jet, b) lobed jet 1, c) lobed jet 2 .

Table 2. Exit conditions for the results presented in Figures 7, 2a-c.

\begin{tabular}{cccccccccc}
\hline $\begin{array}{c}\text { Nozzle } \\
\text { geometry }\end{array}$ & $Q_{0}\left(\mathrm{~m}^{3} \cdot \mathrm{s}^{-1}\right)$ & $U_{0}\left(\mathrm{~m}_{\mathrm{s}}{ }^{-1}\right)$ & $U_{0 \mathrm{C}}\left(\mathrm{m}_{\mathrm{s}}{ }^{-1}\right)$ & $R e_{0}$ & $R e_{0 \mathrm{C}}$ & $\theta_{0}(\mathrm{~mm})$ & \multicolumn{2}{c}{$f_{n}(\mathrm{~Hz})$} \\
\hline Circular & $1.3010^{-3}$ & 1.04 & 1.32 & 2655 & 3372 & 0.945 & & 24 \\
6-lobe 1 & $1.2010^{-3}$ & 0.96 & 1.52 & 2455 & 3873 & 0.84 & 0.46 & 40 & 50 \\
6-lobe 2 & $1.1110^{-3}$ & 0.89 & 1.59 & 2267 & 4051 & 1.68 & 0.51 & 83 & 112 \\
\hline
\end{tabular}

This way, the streamwise structures appearing at the discontinuity locations of the azimuthal structures are not attenuated by their shedding. This lets us suggest that the discontinuity of the azimuthal structures in the lobed jet participates at the mixing enhancement phenomenon.

While the transverse shear of the lobed jet is amplified by the lobe peaks and troughs inclination angles (Fig. 5c), the instantaneous streamwise vorticity field $\omega_{X}=\frac{D_{\mathrm{e}}}{D_{0 \mathrm{c}}}\left(\frac{1}{r} u_{\phi}-\frac{\partial u_{\phi}}{\partial r}-\frac{1}{r} \frac{\partial u_{r}}{\partial \phi}\right)$ is organized in large scale structures of high magnitude levels (Figs. 9, 2c), result that was previously observed by $\mathrm{Hu}$ et al. [10].

As shown in Figures 9, 3c, in the lobed jet 2 the instantaneous fields of the streamwise vorticity and of the transverse shear $\sigma_{r \phi}=\frac{1}{2} \frac{D_{\mathrm{e}}}{U_{0 c}}\left(-\frac{1}{r} u_{\phi}+\frac{\partial u_{\phi}}{\partial r}+\frac{1}{r} \frac{\partial u_{r}}{\partial \phi}\right)$ are perfectly correlated. Figures 9 , $3 \mathrm{a}$ and $3 \mathrm{~b}$ show that this is not the case for the circular and for the lobed jet 1 . This permits us to conclude that the intensification and organization of the vorticity field in the lobed jet 2 is due 

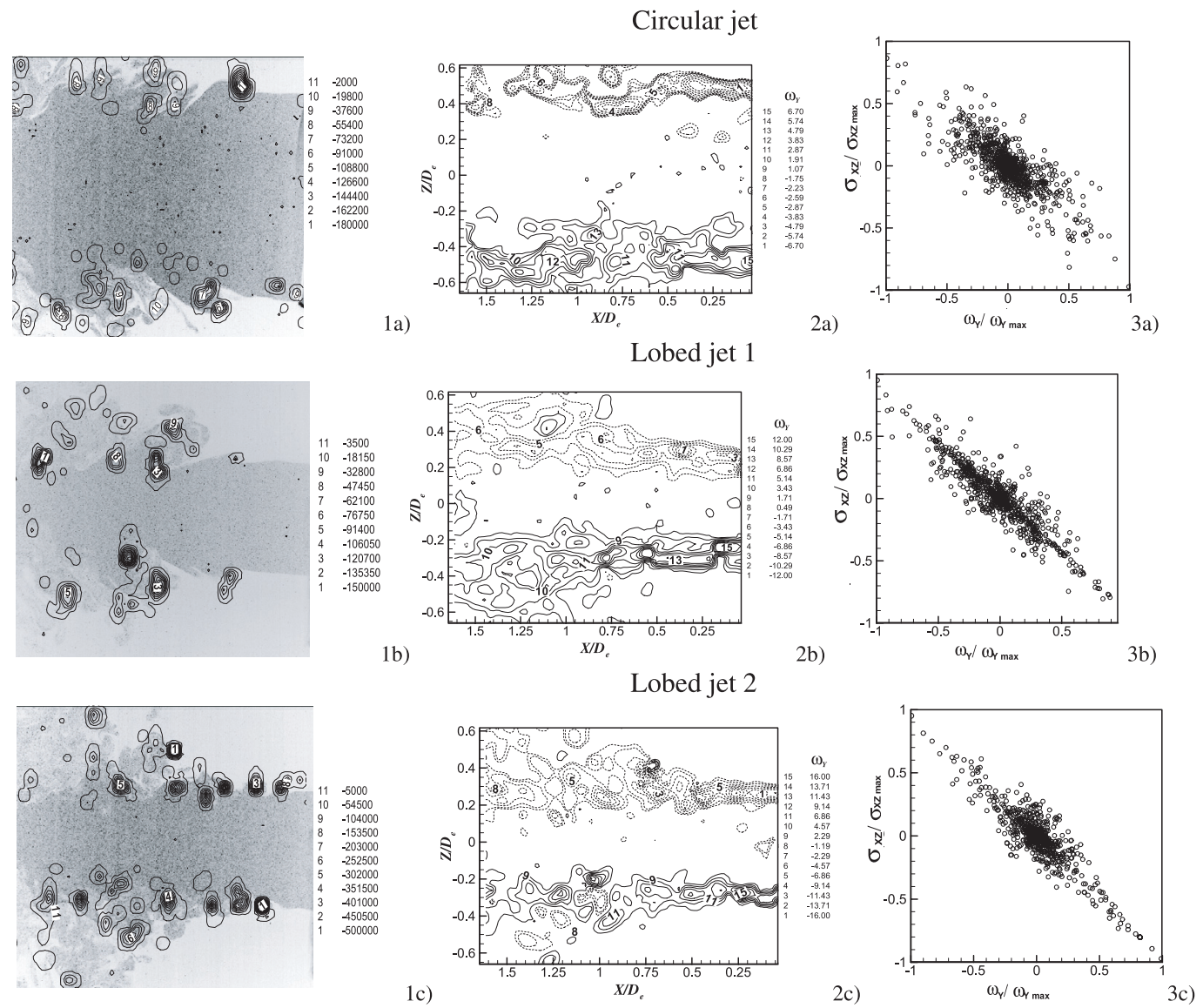

Fig. 8. Instantaneous distributions in the streamwise $(X Z)$ plane: (1) $\lambda_{2}<0$, (2) azimuthal vorticity, (3) Correlation between the in-plane shear and the azimuthal vorticity.

to the shear produced by the lobe peaks and troughs inclinations, leading to a consequent mixing enhancement.

\section{Conclusions}

This study allowed a quantification of the entrainment benefit of two lobed jets compared to a reference circular jet having the same exit area and volumetric flow rate. The two lobed nozzles having the same geometry of the exit plane, set apart by their geometry in the three dimensional space: the first lobed nozzle has no lobe inclinations and the second one has the lobe peaks inclined towards the outer flow and the lobe troughs inclined towards the inner flow. If the entrained flow rate of the first lobed jet does not exceed $70 \%$ of the one entrained in circular reference jet, in the case of the second lobed jet the entrained flow rate reaches up to four times the one entrained in the circular jet.

The peripheral momentum thickness distributions in the transverse exit plane show a larger momentum transfer capability and a stronger shear for the second lobed jet.

The analysis of the correlation between the instantaneous fields of the in-plane streamwise shear and the out of plane azimuthal vorticity, shows that the azimuthal structures are generated in all three jets by the shear between the flow and its ambient. The existence of these structures was supported by an objective detection method, based on the identification of the local pressure minima positions in the plane excepted the ones due to the viscous and irrotational strain effects. If in the circular jet these structures are annular, in the lobed jets they organize in "ring sections" having different frequencies whether they are shed at the lobe sides or in the lobe troughs. These discontinuities which favour the development of the streamwise structures playing a major role in the mixing phenomenon, are probably explaining the induction benefit in the lobed jet issued from the nozzle without inclination angles.

In the jet flow issued from the lobed nozzle having an inclination of the lobes peaks and troughs, these discontinuities of the azimuthal structures are associated to an intensification of the streamwise vorticity fields as well as to its organisation in pairs of large scale structures. For this jet the instantaneous fields of the streamwise vorticity and of the in plane shear were found to be perfectly correlated while for the circular and the lobed jet without inclination angles there was no visible correlation found. These results allow concluding that the important benefit in terms of mixing enhancement observed in the case of the second lobed jet owes to the presence of particular 

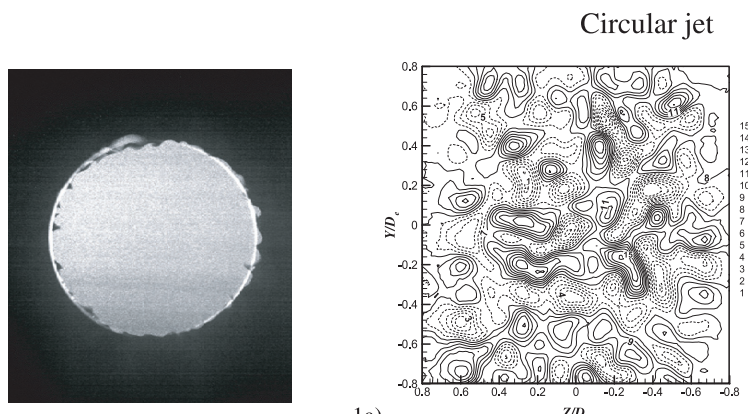

1a)
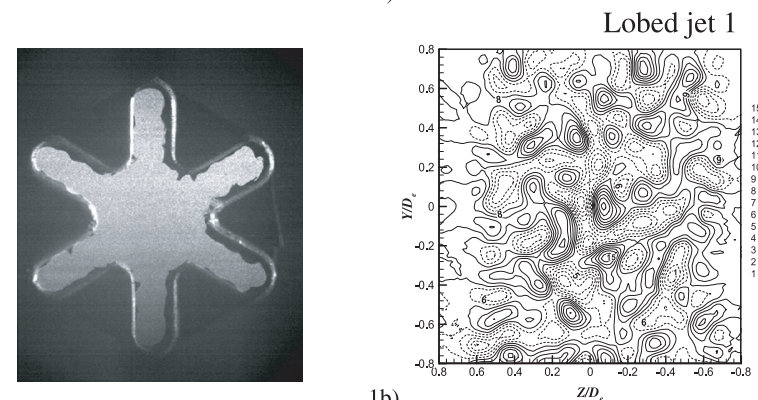

1b)
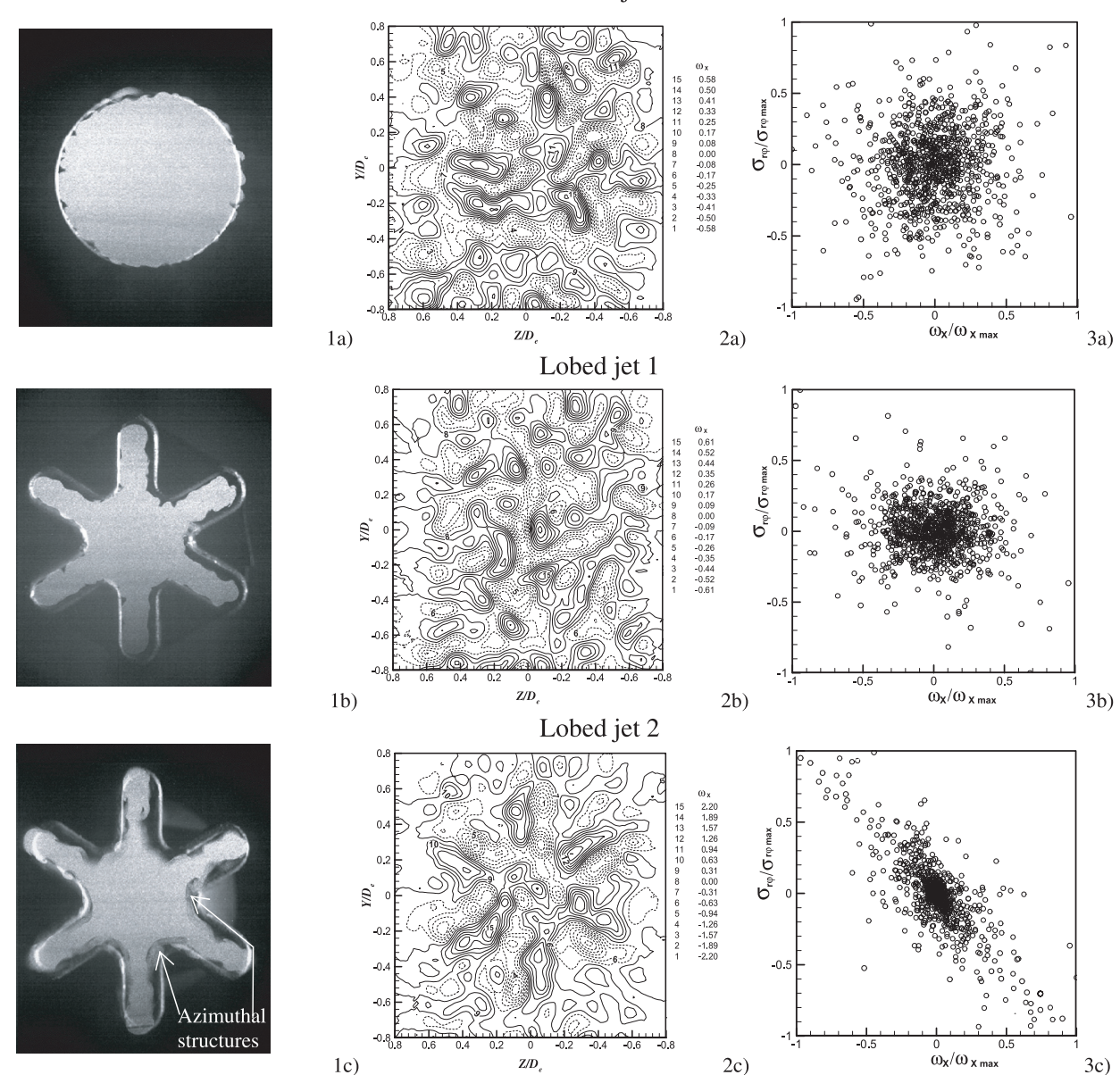

2b)

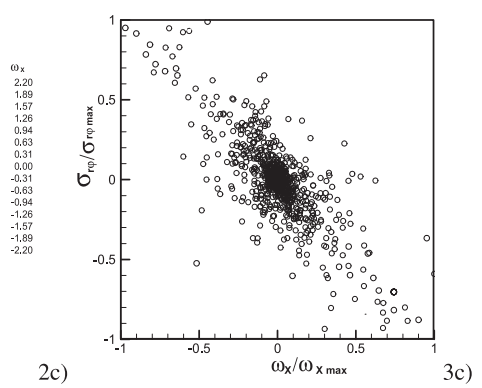

Fig. 9. Transverse $(Y Z)$ plane at $X=0.25 D_{\mathrm{e}}$ : (1) PIV images, (2) instantaneous streamwise vorticity fields, (3) correlation between the in-plane shear and the streamwise vorticity instantaneous fields.

large-scale streamwise structures. These ones are due to the transverse shear amplification induced by the lobe peaks and troughs inclinations.

\section{References}

[1] K.B.M.Q. Zaman, A.K.M.F. Hussain, Vortex pairing in a circular jet under controlled excitation, Part 1, General response, J. Fluid Mech. 101 (1980) 449-441

[2] F. Hussain, H.S. Hussain, Elliptic jets, Part 1, Characteristics of unexcited and excited jets, J. Fluid Mech. 208 (1989) 257-320

[3] Y.T. Lin, M.S. Shich, H.D. Liou, C.S. Hou, Investigation on the mass entrainment of an acoustically controlled elliptic jet, Inter. Comm. Heat and Mass Transfer 25 (1998) 379-388

[4] A.A. Sfeir, Investigation of three-dimensional turbulent rectangular jets, AIAA J. 17 (1979) 1055-1060

[5] W.R. Quinn, Development of a large-aspect-ratio rectangular turbulent free jet, AIAA J. 32 (1994) 547-554

[6] C.M. Ho, E. Gutmark, Vortex induction and mass entrainment in a small-aspect-ratio elliptic jet, J. Fluid Mech. 179 (1987) 383-405

[7] K.B.M.Q. Zaman, Spreading characteristics and thrust of jets from asymmetric nozzles, AIAA (1996) 1-17
[8] H. Hu, T. Kobayashi, S. Wu, G. Shen, Changes to the vortical and turbulent structure of jet flows due to mechanical tabs, Proc. Instn. Mech. Engrs. 213 (1999) 321329

[9] V.M. Belovich, M. Samimy, Mixing processes in a coaxial geometry with a central lobed mixer-nozzle, AIAA J. 35 (1997) 838-841

[10] H. Hu, T. Kobayashi, T. Saga, S. Sagawa, N. Taniguchi, Particle image velocimetry and planar laser-induced fluorescence measurements on lobed jet mixing flows, Experiments in Fluids (2000) S141-S157

[11] H. Hu, T. Saga, T. Kobayashi, N. Taniguchi, Research on the vortical and turbulent structures in the lobed jet flow using laser induced fluorescence and particle image velocimetry techniques, Measurement Science and Technology 11 (2000) 698-711

[12] A.K.M.F. Hussain, K.B.M.Q. Zaman, The preferred mode of the axisymmetric jet, J. Fluid Mech. 110 (1981) $39-71$

[13] J. Jeong, F. Hussain, On the identification of a vortex, J. Fluid Mech. 285 (1995) 69-94

[14] D. Liepmann and M. Gharib, The role of streamwise vorticity in the near-field entrainment of round jets, J. Fluid Mech. 245 (1992) 643-668 